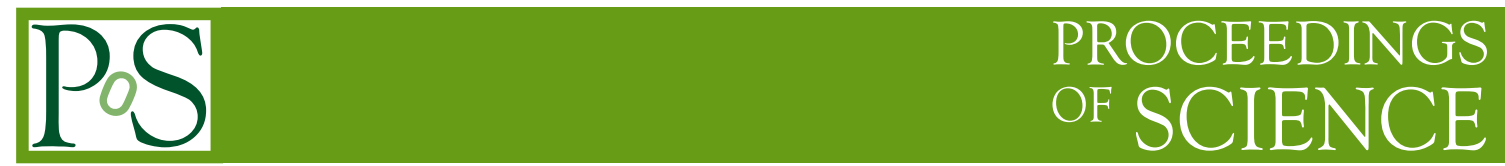

\title{
Moduli Stabilization and Phenomenology
}

\author{
Emilian Dudas* \\ CPhT, Ecole Polytechnique, 91128 Palaiseau Cedex, France \\ LPT, Bat. 210, Univ. Paris-Sud, F-91405 Orsay, France \\ E-mail: emilian.dudasecpht.polytechnique.fr
}

We use the F-term dynamical supersymmetry breaking models with metastable vacua in order to uplift the vacuum energy in the KKLT moduli stabilization scenario. The main advantage compared to earlier proposals, based on D-term contributions, is the manifest supersymmetric treatment and the natural coexistence of a $\mathrm{TeV}$ gravitino mass with a zero cosmological constant. We discuss the structure of soft terms in the observable sector.

From Strings to $\mathrm{LHC}$

January 2-10, 2007

Goa, India

*Speaker. 


\section{Introduction and Conclusions}

In the last couple of years, convincing models of moduli stabilization in string theory were proposed, the propotype being the KKLT scenario [1], based on the orientifolds of IIB string theory flux compactifications [2]. One of the main problems of the KKLT scenario is the uplift of the vacuum energy to zero or positive values. The original proposal of using antibranes relies essentially on nonlinearly realized supersymmetry, whereas the latter attempts $[3,4]$ to uplift vacuum energy by D-terms, based on the suggestion in [5], lead generically to very heavy (close to the Planck mass) gravitino mass. Alternative uplifting using F-terms were already studied in $[7,8,9]$. As already stressed in [8], a generic F-type supersymmetry breaking with a supersymmetry breaking scale $\mathrm{TeV} \ll \Lambda_{\mathrm{SUSY}} \ll M_{\mathrm{Pl}}$ can naturally produce the appropriate, intermediate energy scale, for an uplift with a gravitino mass in the $\mathrm{TeV}$ range. Dynamical supersymmetry breaking is certainly the best candidate to fulfill this criterion.

Recently, Intriligator, Seiberg and Shih (ISS) proposed a simple, vector-like model with longlived, metastable supersymmetry breaking vacua [6], whereas the ground state is supersymmetric. Metastable vacua have by definition a positive contribution to the vacuum energy which could clearly realize the uplifting required in the KKLT scenario [10]. As we will see in this talk, dynamical supersymmetry breaking in metastable vacua of the ISS type does achieve the goal of uplifting the KKLT vacuum energy to zero, while keeping a $\mathrm{TeV}$ scale gravitino mass and therefore leading to low energy supersymmetry. We would like to emphasize, however, that the main ingredient in realizing the uplifting is not the metastable nature of the ISS model.

It is of great interest to couple the Minimal Supersymmetric Standard Model to our present ISS-KKLT setup, to work out the low-energy phenomenology of the model and to compare it to the existing works [11] based on the original KKLT uplifting prescription relying on antibranes and nonlinearly realized supersymmetry.

The structure of this talk is as follows. In Section 2 we combine the KKLT model of moduli stabilization in type IIB strings with the ISS model of metastable supersymmetry breaking vacuum. We show that in this case the uplifting of the vacuum energy is naturally compatible with a $\mathrm{TeV}$ gravitino mass. We discuss the effects of gauging the color symmetry in the ISS model and the lifetime of the metastable vacuum. In Section 3 we provide some general comments about the treelevel soft masses. We then apply the general formulæ for the specific case of the model defined in Section 2 and work out some tree-level soft terms, showing that generically tree-level soft masses are of the order of the gravitino mass, whereas gaugino masses can be suppressed in particular cases.

\section{Metastable vacua and moduli stabilization}

The model is defined by

$$
\begin{aligned}
& W=W_{1}(T)+W_{2}\left(\chi^{i}\right) \\
& K=-3 \ln (T+\bar{T})+|\varphi|^{2}+|\tilde{\varphi}|^{2}+|\Phi|^{2} .
\end{aligned}
$$


In (2.1), $\chi^{i}$ denotes collectively the fields $\varphi_{i}^{a}, \tilde{\varphi}_{a}^{\bar{j}}, \Phi_{\bar{j}}^{i}$ of the ISS model, where $i, \bar{j}=1 \cdots N_{f}$ are flavor indices and $a, b=1 \cdots N$ are color indices. Moreover, in (2.1)

$$
\begin{aligned}
& W_{1}(T)=W_{0}+a e^{-b T}, \\
& W_{2}\left(\chi^{i}\right)=h \operatorname{Tr} \tilde{\varphi} \Phi \varphi-h \mu^{2} \operatorname{Tr} \Phi .
\end{aligned}
$$

Notice that the model is a straightforward combination of the ISS model of metastable supersymmetry breaking vacua with the KKLT model of moduli stabilization. As explained in [6], the sector $\varphi_{i}^{a}, \tilde{\varphi}_{a}^{\bar{j}}$ has a perturbative description in the free magnetic range $N_{f}>3 N$. An appropriate microscopic theory justifying the action (2.2 is described in [10].

As transparent in (2.1), the KKLT and the ISS sectors are only coupled through gravitational interactions. In particular, as the ISS gauge group comes from D3 branes, the dynamical scale in the electric theory and therefore also the mass parameter $\mu$ in the magnetic theory superpotential (2.2) depend on the dilaton $S$, which we assume is already stabilized by NS-NS and RR threeform fluxes. We believe this decoupling is instrumental in getting the uplift of the vacuum energy. Another reason for forbidding a coupling of the dynamical supersymmetry breaking sector in the global supersymmetric limit to the $T$-modulus is that it is unclear how to formulate the non-abelian Seiberg duality for field-dependent couplings.

At the global supersymmetry level and before gauging the color symmetry, the ISS model has a global symmetry $G=S U(N) \times S U\left(N_{f}\right) \times S U\left(N_{f}\right) \times U(1)_{B} \times U(1)^{\prime} \times U(1)_{R}$, broken explicitly to $S U(N) \times S U\left(N_{f}\right) \times U(1)_{B} \times U(1)_{R}$ by the mass parameter $\mu$. In the supergravity embedding (2.2), the R-symmetry $U(1)_{R}$ is explicitly broken. To start with, we consider the ungauged theory, in which the $S U(N)$ is part of the global symmetry group. At the global supersymmetry level, the metastable ISS vacuum is

$$
\Phi_{0}=0 \quad, \quad \varphi_{0}=\tilde{\varphi}_{0}^{T}=\left(\begin{array}{c}
\mu I_{N} \\
0
\end{array}\right)
$$

where $I_{N}$ is the $N \times N$ identity matrix and $\mu \ll \Lambda_{m}$, where $\Lambda_{m} \leq M_{\mathrm{Pl}}$ denotes the mass scale associated with the Landau pole for the gauge coupling in the magnetic theory. The first question to address is the vacuum structure of the model. In order to answer this question, we start from the supergravity scalar potential

$$
V=e^{K}\left[\left(K^{-1}\right)^{i \bar{j}} D_{i} W D_{\bar{j}} \bar{W}-3|W|^{2}\right]+\frac{1}{2}\left(\operatorname{Re} f_{a}\right) D_{a}^{2},
$$

where $\operatorname{Re} f_{a}=1 / g_{a}^{2}$ define the gauge couplings. By using ${ }^{1}(2.1)-(2.2)$, we find

$$
V=\frac{e^{\bar{\chi}_{i} \chi^{i}}}{(T+\bar{T})^{3}}\left\{\frac{(T+\bar{T})^{2}}{3}\left|\partial_{T} W-\frac{3}{T+\bar{T}} W\right|^{2}+\sum_{i}\left|\partial_{i} W+\bar{\chi}_{\bar{i}} W\right|^{2}-3|W|^{2}\right\} .
$$

Since $\mu \ll M_{\mathrm{Pl}}$, the vev's in the ISS model are well below the Planck scale. Then an illuminating way of rewriting the scalar potential (2.5) is to expand it in powers of the fields $\chi^{i} / M_{\mathrm{Pl}}$, in

\footnotetext{
${ }^{1}$ The gauge D-term contributions do not exist in the ungauged case we are discussing in this section and will play essentially no role in the following sections.
} 
which case it reads ${ }^{2}$

$$
\begin{aligned}
& V=\frac{1}{(T+\bar{T})^{3}} V_{\mathrm{ISS}}\left(\chi^{i}, \bar{\chi}_{\bar{i}}\right)+V_{\mathrm{KKLT}}(T, \bar{T})+\frac{\bar{\chi}_{\bar{i}} \chi^{i}}{M_{\mathrm{Pl}}^{2}} V_{1}(T, \bar{T}) \\
& +\frac{1}{M_{\mathrm{Pl}}^{3}}\left[W_{2}\left(\chi^{i}\right) V_{2}(T, \bar{T})+\chi^{i} \partial_{i} W_{2} V_{3}(T, \bar{T})+\text { h.c. }\right]+\cdots,
\end{aligned}
$$

where by comparing (2.6) with (2.5) we can check that $V_{1} \sim m_{3 / 2}^{2} M_{\mathrm{Pl}}^{2}$, and $V_{2}, V_{3} \sim m_{3 / 2} M_{\mathrm{Pl}}^{3}$, where as usual $m_{3 / 2}^{2}=|W|^{2} \exp (K)$. Notice that the contribution to the vacuum energy from the ISS sector, in the global limit, is

$$
\left\langle V_{\text {ISS }}\right\rangle=\left(N_{f}-N\right) h^{2} \mu^{4} .
$$

Since we are interested in small ( $\mathrm{TeV}$ scale) gravitino mass, it is clear that the first two terms in the rhs of (2.6), $V_{\mathrm{ISS}}$ and $V_{\mathrm{KKLT}}$, are the leading terms. Consequently, there should be a vacuum very close to an uplift KKLT vacuum $\langle T\rangle=T_{0}$ and the ISS vacuum $\left\langle\chi^{i}\right\rangle=\chi_{0}^{i}$. The KKLT uplift vacuum at the zeroth order $T_{0}$ is defined as the minimum of the zeroth order potential $\partial_{T_{0}} V_{0}=0$, obtained by inserting the ISS vacuum (2.3) into the supergravity scalar potential

$$
V_{0}=\frac{1}{(T+\bar{T})^{3}}\left[\frac{(T+\bar{T})^{2}}{3}\left|D_{T} W_{1}\right|^{2}-3\left|W_{1}\right|^{2}+h^{2}\left(N_{f}-N\right) \mu^{4}\right] .
$$

In the limit $b T \gg 1$ and for zero cosmological constant, a good approximation for $T_{0}$, considered to be real in what follows, is provided by

$$
W_{0}+\frac{a b\left(T_{0}+\bar{T}_{0}\right)}{3} e^{-b T_{0}}=0 .
$$

Notice that in this case $T$ does contribute to supersymmetry breaking ${ }^{3}$

$$
F^{T} \equiv e^{\frac{K}{2}} K^{T \bar{T}} \overline{D_{T} W} \simeq \frac{a}{\left(T_{0}+\bar{T}_{0}\right)^{1 / 2}} e^{-b T_{0}}
$$

but by an amount suppressed by a factor of $1 / b\left(T_{0}+\bar{T}_{0}\right)$ compared to the naive expectation.

The cosmological constant at the lowest order is given by

$$
\Lambda=V_{\mathrm{KKLT}}\left(T_{0}, \bar{T}_{0}\right)+\frac{\left(N_{f}-N\right) h^{2} \mu^{4}}{\left(T_{0}+\bar{T}_{0}\right)^{3}},
$$

which shows that the ISS sector plays the role of an uplifting sector of the KKLT model. In the zeroth order approximation and in the large volume limit $b\left(T_{0}+\bar{T}_{0}\right) \gg 1$, we find that the condition of zero cosmological constant $\Lambda=0$ implies roughly

$$
3\left|W_{0}\right|^{2} \sim h^{2}\left(N_{f}-N\right) \mu^{4} .
$$

If we want to have a gravitino mass $m_{3 / 2} \sim W_{0} /\left(T_{0}+\bar{T}_{0}\right)^{3 / 2}$ in the TeV range, we need small values of $\mu \sim 10^{-6}-10^{-7}$. Since $\mu$ in the model [6] has a dynamical origin, this is natural. Moreover,

\footnotetext{
${ }^{2}$ In most of the formulæ of this letter, $M_{\mathrm{Pl}}=1$. In some formulæ, however, we keep explicitly $M_{\mathrm{Pl}}$.

${ }^{3}$ Notice that the leading order expression for $W_{0}$ in (2.9) is not enough for computing $F^{T}$, since the subleading terms neglected in (2.9) are needed as well. $F^{T}$ can be computed directly, however, by keeping the leading terms in the eq. $\partial_{T} V=0$.
} 
the metastable vacuum of [6] has a significantly large lifetime exactly in this limit, more precisely when $\varepsilon \equiv\left(\mu / \Lambda_{m}\right) \ll 1$. Therefore, a light (TeV range) gravitino mass is natural in our model and compatible with the uplift of the cosmological constant. We believe that this fact is an improvement over the D-term uplift models suggested in [5] and worked out in [4].

Notice that supergravity corrections give tree-level masses to the pseudo-moduli fields of the ISS model. As explained in more general terms in [6], these corrections are subleading with respect to masses arising from the one-loop Coleman-Weinberg effective potential in the global supersymmetric limit. This can be explicitly checked starting from the supergravity scalar potential (2.5) and expanding in small fluctuations around the vacuum (2.3) to the quadratic order.

\subsection{Gauging the model : infrared description}

In the ISS model, the $S U(N)$ symmetry is gauged and corresponds to the gauge group of the magnetic theory. In the electric description, the ISS model is the supersymmetric QCD with $N_{c}$ colors and $N_{c}<N_{f}<3 N_{c} / 2$ quark flavors $Q, \tilde{Q}$ such that in the magnetic description with the gauge group $S U\left(N_{f}-N_{c}\right)$, the number of flavors is large $N_{f}>3 N$, where the magnetic theory is in the infrared-free phase. In this case the perturbative magnetic description, around the origin in field space, is reliable. The electric theory has a dynamical scale $\Lambda$ and a mass term for the quarks $W=m_{i}^{\bar{j}} Q^{i} \tilde{Q}_{\bar{j}}$. There are $N_{c}$ vacua described by

$$
M_{\bar{j}}^{i}=\left(\frac{1}{m}\right)_{\bar{j}}^{i}(\operatorname{det} m)^{\frac{1}{N_{c}}} \Lambda^{\frac{3 N_{c}-N_{f}}{N_{c}}} .
$$

The perturbative treatment in the magnetic description translates into the constraint $m_{a} \ll \Lambda$, where $a$ denotes here the number of light mass eigenvalues, which has to be equal or larger to $N_{f}+1$ in order for the metastable vacua to exist.

Denoting by $\Lambda_{m}$ the Landau pole of the magnetic theory, according to ISS, for arbitrary vev's of $\Phi$ the quark flavors become massive and can be integrated out. By doing this and by coupling the resulting low-energy system to the KKLT model, we get a lagrangian described by

$$
\begin{aligned}
& W=W_{0}+a e^{-b T}+N\left(\frac{h^{N_{f}} \operatorname{det} \Phi}{\Lambda_{m}^{N_{f}}-3 N}\right)^{1 / N}-h \mu^{2} \operatorname{Tr} \Phi \\
& K=-3 \ln (T+\bar{T})+\bar{\Phi} \Phi .
\end{aligned}
$$

Similarly to the global supersymmetry analysis of ISS [6], this action has $N_{f}-N$ supersymmetric vacua, which in the global limit are given by

$$
\langle h \Phi\rangle=\Lambda_{m} \varepsilon^{2 N /\left(N_{f}-N\right)} I_{N_{f}}=\mu \frac{1}{\varepsilon^{\left(N_{f}-3 N\right) /\left(N_{f}-N\right)}} I_{N_{f}},
$$

where $\varepsilon \equiv \mu / \Lambda_{m}$. The vacuum in the $T$-direction is simpler to describe by replacing the vev's (2.15) in the superpotential (2.14). By doing this, we get an effective superpotential

$$
W_{\mathrm{eff}}=W_{0}-\frac{\left(N_{f}-N\right) \mu^{3}}{\varepsilon^{\left(N_{f}-3 N\right) /\left(N_{f}-N\right)}}+a e^{-b T} .
$$

Since $W_{0}<0$ in the KKLT model, the effect of the supersymmetric $\Phi$ vev's is to increase the absolute value of the (negative) constant in the superpotential. The values of the minimum for $T$ 
and the corresponding negative cosmological constant are given approximately by

$$
\begin{aligned}
& a b e^{-b T_{s}}+\frac{3}{T_{s}+\bar{T}_{s}}\left(W_{0}-\frac{\left(N_{f}-N\right) \mu^{3}}{\varepsilon^{\left(N_{f}-3 N\right) /\left(N_{f}-N\right)}}\right) \simeq 0, \\
& V_{0} \simeq-\frac{3}{\left(T_{s}+\bar{T}_{s}\right)^{3}}\left|W_{0}-\frac{\left(N_{f}-N\right) \mu^{3}}{\varepsilon^{\left(N_{f}-3 N\right) /\left(N_{f}-N\right)}}\right|^{2} .
\end{aligned}
$$

The supersymmetric ISS vacuum is therefore AdS.

Notice that for $W_{0} \gg \mu^{3} / \varepsilon^{\left(N_{f}-3 N\right) /\left(N_{f}-N\right)}$, we get $T_{s} \sim T_{0}$, with $T_{0}$ defined in (2.9), since in this case $W \simeq W_{0}$. If $W_{0} \ll \mu^{3} / \varepsilon^{\left(N_{f}-3 N\right) /\left(N_{f}-N\right)}$, then $T_{s}<T_{0}$.

\subsection{Lifetime of the metastable vacuum}

The model we discussed in this paper has one metastable vacuum and two type of AdS supersymmetric minima. The metastable vacuum will tunnel to the supersymmetric AdS minimum (2.15)-(2.17). The purpose of this section is to provide a qualitative estimate of the lifetime of the metastable minimum, following [12],[13]. The bounce action is expected to come from the path in field space of minimum potential barrier between the metastable supersymmetry breaking vacuum and the supersymmetric vacua. Along this path, the bounce action cannot be computed analytically. For a triangular idealized approximation [13], the bounce action $S_{b}$ is qualitatively

$$
S_{b} \sim \frac{(\Delta \chi)^{4}}{\Delta V},
$$

where $\Delta V$ is the (minimum) barrier along the bounce and $\Delta \chi$ is the variation of the relevant field. For the tunneling between the metastable ISS vacuum (2.3) and the supersymmetric one (2.15) after gauging $S U(N)$, there are two cases. If $\mu \ll \varepsilon^{\left(N_{f}-3 N\right) /\left(N_{f}-N\right)} M_{\mathrm{Pl}}$, we get

$$
h \Delta \Phi \simeq \mu \frac{1}{\varepsilon^{\left(N_{f}-3 N\right) /\left(N_{f}-N\right)}} \quad, \quad \Delta V \sim \frac{3}{\left(T_{s}+\bar{T}_{s}\right)^{3}}\left|W_{0}\right|^{2} .
$$

Then, by using the condition (2.12) for a zero vacuum energy in the metastable vacuum, we get

$$
S_{b} \sim \frac{\left(T_{s}+\bar{T}_{s}\right)^{3}}{\varepsilon^{4\left(N_{f}-3 N\right) /\left(N_{f}-N\right)}} \gg 1
$$

which increases the lifetime of the metastable vacuum compared to the similar ISS analysis. The reason is that the energy difference between the metastable and the AdS supersymmetric minimum is decreased by the factor $1 /\left(T_{s}+\bar{T}_{s}\right)^{3}$, resulting in an increase in the bounce action $S_{b}$. In the case where $\mu \gg \varepsilon^{\left(N_{f}-3 N\right) /\left(N_{f}-N\right)} M_{\mathrm{Pl}}$, the vacuum energy of the supersymmetric vacuum (2.17) and consequently $\Delta V$ change. The bounce action in this case is

$$
S_{b} \sim \frac{M_{\mathrm{Pl}}^{2}}{\mu^{2}} \frac{\left(T_{s}+\bar{T}_{s}\right)^{3}}{\varepsilon^{2\left(N_{f}-3 N\right) /\left(N_{f}-N\right)}} \gg 1
$$

The metastable minimum could also tunnel to other AdS supersymmetric minima [10]. Even by taking seriously the effective theory analysis in this case, we notice that generically the AdS supersymmetric minima are far away in the $\Phi$ field space from the ISS-KKLT metastable vacuum (2.3), (2.9). The tunneling probability to go to the AdS vacua is highly suppressed and irrelevant for all practical purposes. 


\section{Soft terms and mass scales}

\subsection{General tree-level formulæ}

The relevant couplings for our present discussion are the following terms in the Kähler potential and the superpotential arising from the perturbative expansion in the matter fields $M^{I}$

$$
\begin{aligned}
& K \rightarrow K+\left[(T+\bar{T})^{n_{I}} Z_{I \bar{J}}+\cdots\right] M^{I} \bar{M}^{\bar{J}}+\cdots \equiv K+K_{I \bar{J}} M^{I} \bar{M}^{\bar{J}} \\
& W \rightarrow W+\frac{1}{6} W_{I J K} M^{I} M^{J} M^{K}
\end{aligned}
$$

where $\cdots$ denote couplings to other (hidden-sector, messengers in gauge mediation models, etc) fields. In a manifestly supersymmetric approach, with both F and D-term contributions, the condition of zero cosmological constant is

$$
K_{\alpha \bar{\beta}} F^{\alpha} F^{\bar{\beta}}+\sum_{a}\left(g_{a}^{2} / 2\right) D_{a}^{2}=3 m_{3 / 2}^{2} M_{\mathrm{Pl}}^{2}
$$

where $\alpha, \bar{\beta}$ refer to fields contributing to supersymmetry breaking and $a$ is an index for anomalous $U(1)$ gauge factors. Then the most general formulæ for soft terms of matter fields ${ }^{4} M^{I}\left(F^{I}=0\right)$, are given by [3] for the heterotic strings case)

$$
\begin{aligned}
& m_{I \bar{J}}^{2}=m_{3 / 2}^{2} K_{I \bar{J}}-F^{\alpha} F^{\bar{\beta}} R_{\alpha \bar{\beta} I \bar{J}}-\sum_{a} g_{a}^{2} D_{a}\left(\frac{1}{2} K_{I \bar{J}}-\partial_{I} \partial_{\bar{J}}\right) D_{a} \\
& A_{I J K}=m_{3 / 2}^{2}\left(3 \nabla_{I} \nabla_{J} G_{K}+G^{\alpha} \nabla_{I} \nabla_{J} \nabla_{K} G_{\alpha}\right)-g_{a}^{2} D_{a}\left(\frac{D_{a}}{2} \nabla_{i} \nabla_{j} G_{k}-\nabla_{i} \nabla_{j} \nabla_{k} D_{a}\right), \\
& M_{1 / 2}^{a}=\frac{1}{2}\left(R e f_{a}\right)^{-1} m_{3 / 2} G^{\alpha} \partial_{\alpha} f_{a},
\end{aligned}
$$

where $G=K+\ln |W|^{2}, G_{\alpha}=\partial_{\alpha} G, \nabla_{I} G_{J}=G_{I J}-\Gamma_{I J}^{K} G_{K}$, etc., where $R_{\alpha \bar{\beta} I \bar{J}}=\partial_{\alpha} \partial_{\bar{\beta}} K_{I \bar{J}}-$ $\Gamma_{\alpha I}^{M} K_{M \bar{N}} \Gamma_{\bar{\beta} \bar{J}}^{\bar{N}}$ is the Riemann tensor of the Kähler manifold and $\Gamma_{\alpha I}^{M}=K^{M \bar{N}} \partial_{\alpha} K_{\bar{N} I}$ are the Christoffel symbols. Moreover,

$$
D_{a}=X_{I}^{a} M^{I} \partial_{I} K-\frac{\eta_{a}^{\alpha}}{2} \partial_{\alpha} K .
$$

In (3.4), $X_{I}^{a}$ denote $U(1)_{a}$ charges of charged fields $M^{I}$, and $\eta_{a}^{\alpha}$ are defined by the nonlinear gauge transformations of the moduli fields under (super-)gauge fields transformations

$$
V_{a} \rightarrow V_{a}+\Lambda_{a}+\bar{\Lambda}_{a} \quad, \quad T_{\alpha} \rightarrow T_{\alpha}+\eta_{a}^{\alpha} \Lambda_{a}
$$

By using (3.4), we can also write the scalar masses in (3.3) as

$$
m_{I \bar{J}}^{2}=m_{3 / 2}^{2} K_{I \bar{J}}-F^{\alpha} F^{\bar{\beta}} R_{\alpha \bar{\beta} I \bar{J}}-\sum_{a} g_{a}^{2} D_{a}\left(\frac{1}{2} D_{a}-X_{I}^{a}-v_{l} X_{l}^{a} \partial_{l}+\frac{\eta_{a}^{\alpha}}{2} \partial_{\alpha}\right) K_{I \bar{J}}
$$

where $v_{l}$ are vev's of charged scalar fields $z^{l}$ with charge $X_{l}^{a}$.

\footnotetext{
${ }^{4}$ We don't write the analytic bilinear soft terms, since their discussion depends on the origin of the corresponding ( $\mu$-like) term in the superpotential.
} 


\subsection{Soft terms with dynamical F-term uplifting}

A particularly important question is the magnitude of the soft terms in the visible sector in the present setup. In order to answer this question, we first estimate the contribution to supersymmetry breaking from the various fields. By using the results of section 2, we find in the leading order

$$
\begin{aligned}
& \overline{F^{\varphi}} \equiv e^{K / 2} K^{\varphi \bar{\varphi}} D_{\varphi} W \simeq e^{K / 2} K^{\varphi \bar{\varphi}}\left(\bar{\varphi}_{0} W+\delta \Phi \partial_{\Phi} \partial_{\varphi} W_{2}\right) \simeq 0, \\
& \overline{F^{\tilde{\varphi}}} \simeq 0 \quad, \quad \overline{F^{\Phi}}=e^{K / 2}\left(\begin{array}{cc}
0 & 0 \\
0 & -h \mu^{2} I_{N_{f}-N}
\end{array}\right), \\
& F^{T} \simeq \frac{a}{\left(T_{0}+\bar{T}_{0}\right)^{1 / 2}} e^{-b T_{0}} \simeq-\frac{3}{b} m_{3 / 2} .
\end{aligned}
$$

Notice that the main contribution to supersymmetry breaking comes from the magnetic mesonic fields $\Phi$, which are the main responsible for the uplift of the vacuum energy

$$
\operatorname{Tr}\left(\left|F^{\Phi}\right|^{2}\right) \simeq 3 m_{3 / 2}^{2} .
$$

The transmission of supersymmetry breaking in the observable sector depends on the couplings of the observable fields $M^{I}$ to the SUSY breaking fields $\Phi, T$. The relevant couplings for our present discussion are the following terms in the Kähler metric of the matter fields $M^{I}$

$$
K_{I \bar{J}}=(T+\bar{T})^{n_{I}} Z_{I \bar{J}}+\operatorname{Tr}\left(|\Phi|^{2}\right) Z_{I \bar{J}}^{\prime},
$$

where the form of the $\Phi$ coupling in the Kähler metric is dictated by the diagonal $S U\left(N_{f}\right)$ flavor symmetry left unbroken by the mass parameter $\mu$ in the ISS lagrangian. The Yukawa couplings $W_{I J K}$ could also depend on $T$ and $\Phi$.

Then from (3.3) with no D-term contributions $D_{a}=0$, we find that the $F^{T}$ contribution is subleading by a factor $1 / b^{2}(T+\bar{T})^{2}$ with respect to the other contributions. This has the nice feature that the flavor-dependent $F^{T}$ contribution to scalar soft masses are subleading. The result for the (canonically normalized scalars) soft masses, at the leading order, is then given by

$$
\begin{aligned}
& m_{I \bar{J}}^{2}=m_{3 / 2}^{2} \delta_{I \bar{J}}+\frac{h^{2}\left(N_{f}-N\right) \mu^{4}}{(T+\bar{T})^{3}}\left(K^{-1} Z^{\prime}\right)_{I \bar{J}} \\
& \simeq m_{3 / 2}^{2}\left(\delta_{I \bar{J}}+3\left(K^{-1} Z^{\prime}\right)_{I \bar{J}}\right) .
\end{aligned}
$$

If the coupling to the mesonic fields $\Phi$ is small, i.e the coefficients $Z_{I \bar{J}}^{\prime}$ are suppressed, soft scalar masses in the observable (MSSM) sector are universal and are similar with the ones obtained in the "dilaton-dominated" scenario in the past. It would be very interesting to find physical reasons of why $Z_{I J}^{\prime}$ are small. The geometrical sequestering cannot be invoked in this case, since the matter fields $M$ and the mesons $\Phi$ do not fit into the peculiar sequestering structure. If the coeff. $Z_{I \bar{J}}^{\prime}$ are of order one, the two terms in (3.10) are of the same order and the flavor problem of gravity mediation is back.

A similar conclusion holds for the other possible source of flavor violation, the A-terms. If the couplings of the mesons to the matter fields are small, we get at the leading order, for the canonically normalized scalars

$$
A_{I J L} \simeq 3 m_{3 / 2} w_{I J L},
$$


where $w_{I J L}$ are the low-energy Yukawa couplings for the matter fields, related to the corresponding SUGRA couplings $W_{I J L}=\nabla_{I} \nabla_{J} \nabla_{L} W$ by

$$
w_{I J L}=e^{K / 2}\left(K^{-1 / 2}\right)_{I}^{I^{\prime}}\left(K^{-1 / 2}\right)_{J}^{J^{\prime}}\left(K^{-1 / 2}\right)_{L}^{L^{\prime}} W_{I^{\prime} J^{\prime} L^{\prime}} .
$$

Since A-terms are proportional to the Yukawa couplings, there are no flavor violations in this case.

Gaugino masses in the observable sector are determined by the gauge kinetic functions which in our case have generically the form

$$
f_{a}=f_{a}^{(0)}+\alpha_{a} T+\beta_{a}(\operatorname{Tr} \Phi),
$$

where $f_{a}^{(0)}$ are provided by other moduli fields, stabilized in a supersymmetric manner. The form of coupling to the mesons in (3.13) is fixed by the diagonal $S U\left(N_{f}\right)$ flavor symmetry left unbroken by the mass parameter $\mu$, whereas $\alpha_{a}$ are numbers of order one ${ }^{5}$. The gaugino masses

$$
M_{a}=\alpha_{a} F^{T}+\beta_{a}\left(\operatorname{Tr} F^{\Phi}\right)
$$

are of the order of the gravitino mass if $\beta_{a}$ are of order one, whereas they are suppressed by the factor $1 / b(T+\bar{T})$ if $\beta_{a}$ are small. In this second case, the anomaly-mediated contributions are comparable to the tree-level ones. To conclude, we do not find a suppression of all the soft terms in the observable sector with respect to the gravitino mass. Therefore our results point towards a gravity-mediation type of supersymmetry breaking in the hidden sector, which in the case of small couplings of matter to hidden sector mesons are very similar to the dilaton-domination scenario and are therefore flavor blind at tree-level ${ }^{6}$.

We would like to briefly compare these results to the ones obtained in [11] by using the original KKLT uplifting mechanism with $\overline{D 3}$ antibranes. By using a nonlinear supergravity approach, [11] found a (moderate) hierarchy $m_{3 / 2} \sim 4 \pi^{2} m_{\text {soft }}$. Let us try to understand better the difference with our results. As we discussed in the previous section, there are three ways of suppressing the treelevel soft masses for matter fields. The first is no-scale type models. The KKLT-type models are not of this type, since the $F^{T}$ contribution is small. The second case is the dominant D-term breaking. This is probably the manifestly supersymmetric case which should correspond in the low energy limit to the analysis done in [11]. Knowing that pure D-term supersymmetry breaking does not exist, it could be difficult to realize a model along these lines. It is however very interesting to investigate this possibility in more detail.

We believe that a more detailed phenomenological analysis of the possible manifestly supersymmetric uplifting mechanisms deserves further investigation.

\section{Acknowledgments}

I would like to thank the organizers of the Goa meeting for organizing a very sucessful meeting and Y. Mambrini, C. Papineau and S. Pokorski for the enjoyable collaborations on which the present talk is based. Work partially supported by the CNRS PICS \# 2530 and 3059, RTN contracts MRTNCT-2004-005104 and MRTN-CT-2004-503369, the European Union Excellence Grant, MEXT-CT2003-509661 and the EC contract MTKD-CT-2005-029466.

\footnotetext{
${ }^{5}$ In a type IIB orientifold embedding, this happens if the observable sector lives on D7 branes.

${ }^{6}$ For other ways of getting fhvor universality in compactifi cations with stabilized moduli, see e.g. [16].
} 


\section{References}

[1] S. Kachru, R. Kallosh, A. Linde and S. P. Trivedi, Phys. Rev. D 68 (2003) 046005 [arXiv:hep-th/0301240].

[2] S. B. Giddings, S. Kachru and J. Polchinski, Phys. Rev. D 66 (2002) 106006 [arXiv:hep-th/0105097].

[3] E. Dudas and S. K. Vempati, Nucl. Phys. B 727 (2005) 139 [arXiv:hep-th/0506172].

[4] G. Villadoro and F. Zwirner, Phys. Rev. Lett. 95 (2005) 231602 [arXiv:hep-th/0508167];

A. Achucarro, B. de Carlos, J. A. Casas and L. Doplicher, arXiv:hep-th/0601190; K. Choi and K. S. Jeong, arXiv:hep-th/0605108; E. Dudas and Y. Mambrini, arXiv:hep-th/0607077.

[5] C. P. Burgess, R. Kallosh and F. Quevedo, JHEP 0310 (2003) 056 [arXiv:hep-th/0309187].

[6] K. Intriligator, N. Seiberg and D. Shih, JHEP 0604 (2006) 021 [arXiv:hep-th/0602239].

[7] A. Saltman and E. Silverstein, JHEP 0411 (2004) 066 [arXiv:hep-th/0402135].

[8] M. Gomez-Reino and C. A. Scrucca, JHEP 0605 (2006) 015 [arXiv:hep-th/0602246] and arXiv:hep-th/0606273.

[9] O. Lebedev, H. P. Nilles and M. Ratz, Phys. Lett. B 636 (2006) 126 [arXiv:hep-th/0603047].

[10] E. Dudas, C. Papineau and S. Pokorski, JHEP 0702 (2007) 028 [arXiv:hep-th/0610297] ; H. Abe, T. Higaki, T. Kobayashi and Y. Omura, Phys. Rev. D 75 (2007) 025019 [arXiv:hep-th/0611024].

[11] K. Choi, A. Falkowski, H. P. Nilles, M. Olechowski and S. Pokorski, JHEP 0411 (2004) 076 [arXiv:hep-th/0411066]; K. Choi, A. Falkowski, H. P. Nilles and M. Olechowski, Nucl. Phys. B 718 (2005) 113 [arXiv:hep-th/0503216]; A. Falkowski, O. Lebedev and Y. Mambrini, JHEP 0511 (2005) 034 [arXiv:hep-ph/0507110].

[12] S. R. Coleman, Phys. Rev. D 15 (1977) 2929 [Erratum-ibid. D 16 (1977) 1248].

[13] M. J. Duncan and L. G. Jensen, Phys. Lett. B 291 (1992) 109.

[14] S. K. Soni and H. A. Weldon, Phys. Lett. B 126 (1983) 215; V. S. Kaplunovsky and J. Louis, Phys. Lett. B 306 (1993) 269 [arXiv:hep-th/9303040]; A. Brignole, L. E. Ibanez and C. Munoz, Nucl. Phys. B 422 (1994) 125 [Erratum-ibid. B 436 (1995) 747] [arXiv:hep-ph/9308271].

[15] P. Binetruy and E. Dudas, Phys. Lett. B 389 (1996) 503 [arXiv:hep-th/9607172]; N. Arkani-Hamed, M. Dine and S. P. Martin, Phys. Lett. B 431 (1998) 329 [arXiv:hep-ph/9803432].

[16] J. P. Conlon, S. S. Abdussalam, F. Quevedo and K. Suruliz, "Soft SUSY breaking terms for chiral matter in IIB string arXiv:hep-th/0610129. 